\title{
Surgical Treatment of Tracheal Lipoma after Multiple Bronchoscopy Interventions and Placements of a Tracheal Stent
}

\author{
Xiaoming Zhang ${ }^{1}$ Tao $\mathrm{ji}^{2}$ Longhai Yang ${ }^{1}$ Yi Liu ${ }^{1}$ Hongsheng Lin ${ }^{1}$ Huiyu Pan ${ }^{1}$ Zizi Zhou ${ }^{1,3}$ \\ ${ }^{1}$ Department of Cardiothoracic Surgery, Shenzhen University General \\ Hospital, Shenzhen, China \\ 2 Department of Cardiothoracic Surgery, Wuhan General Hospital of \\ Guangzhou Military Command, Wuhan, China \\ ${ }^{3}$ Department of Plastic and Reconstructive Surgery, BG Unfallklinik \\ Ludwigshafen, University of Heidelberg, Heidelberg, Germany \\ Thorac Cardiovasc Surg Rep 2019;8:e20-e23. \\ Address for correspondence Zizi Zhou, MD, Department of \\ Cardiothoracic Surgery, Shenzhen University General Hospital, \\ Xueyuan Road No.1098, Xili University Town, 518055 Shenzhen, \\ China (e-mail: zizi.zhou3@gmail.com).
}

Background The majority of adult primary tracheal tumors are malignant; however, as one type of benign tumors, lipoma is extremely uncommon.

Case Description We report a case where lipoma was first misdiagnosed as bronchial asthma, followed by sudden aggravation of dyspnea after trauma, and computed tomography (CT) examination of the neck and chest confirmed a tracheal tumor. Through multiple bronchoscopy interventions and placements of a tracheal stent, little therapeutic benefit was discovered, and resection of the tracheal tumor combined with tracheal end-to-end anastomosis was performed to ultimately achieve a cure.

Conclusion Primary tracheal tumors should be highly suspected in patients with recurrent and gradually worsening dyspnea; timely cervical, thoracic $\mathrm{CT}$ and bronchoscopy can provide an accurate diagnosis. Surgical radical resection is the only way to cure all benign tracheal tumors such as lipoma.

\section{Introduction}

Primary tumors of the trachea account for less than $0.1 \%$ of tumors and most of them are malignant. ${ }^{1}$ As one type of benign tumors, primary tracheal lipoma is very uncommon. Because of the characteristic of slow growth, patients with benign tracheal tumors develop symptoms of airway obstruction gradually. Therefore, they are usually misdiagnosed as asthma, chronic obstructive pulmonary disease, or bronchitis, and the definitive diagnosis of these tumors is usually delayed.

We describe a case where lipoma was first misdiagnosed as bronchial asthma, followed by sudden aggravation of dyspnea after trauma, and cervical, thoracic computed tomography (CT) confirmed a tracheal tumor. Through multiple bronchoscopy interventions and placements of a tracheal stent, little therapeutic benefit was discovered, and resection of the tracheal tumor combined with tracheal end-to-end anastomosis was performed to ultimately achieve a cure.

\section{Case Presentation}

A 35-year-old man has suffered from dry cough and shortness of breath recurrently for 4 years, and those symptoms gradually worsened. During the past 6 months, the patient developed exertional dyspnea after daily activities, accompanied by orthopnea. Therefore, he went to a local hospital many times, and no abnormal sign was found in the chest X-ray, and bronchial asthma was ultimately diagnosed. After treatment with aminophylline, Bricanyl, and aerosol salbutamol, the symptom of dyspnea was alleviated, while it relapsed frequently. After a traffic accident, the patient developed severe dyspnea and could not maintain a supine position; therefore, received

January 29, 2019

accepted

March 22, 2019
DOI https://doi.org/

10.1055/s-0039-1688903. ISSN 2194-7635. (c) 2019 Georg Thieme Verlag KG
Stuttgart · New York

License terms

c) $(1) \$$ 

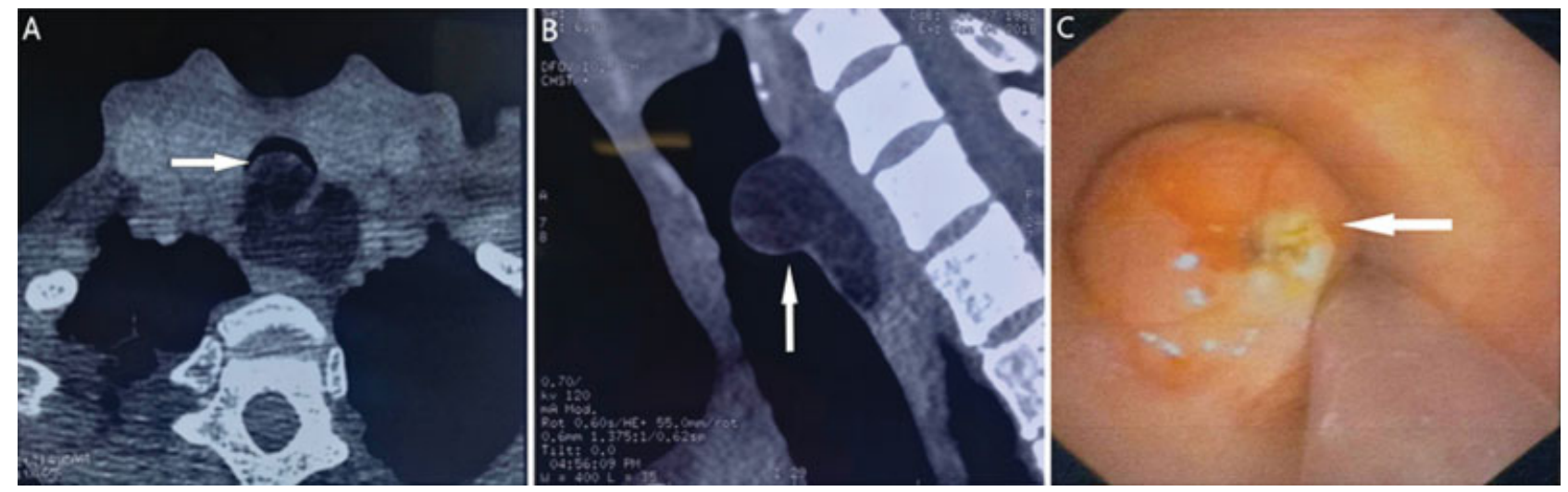

Fig. 1 (A, B) CT showing an intratracheal tumor; (C) bronchoscopy showing a tumor on the posterior wall of the trachea and a fissure-like stenosis between the tumor and tracheal wall. CT, computed tomography.

he went to the local hospital immediately. Physical examination: slight cyanosis of the lips, shortness of breath, obvious three-concave sign during inhalation, skin abrasion on the front chest wall, no thoracic deformity, and wheezing in both lungs, which was more obvious in the upper chest. Arterial blood gas analysis showed a $\mathrm{pH}$ of 7.38, partial pressure of carbon dioxide of $46 \mathrm{~mm} \mathrm{Hg}$, and partial pressure of oxygen of $54 \mathrm{~mm} \mathrm{Hg}$. Chest X-ray showed enhanced light transmission in both lungs and no other abnormalities. CT and three-dimensional (3D) reconstruction of the neck and chest found a soft tissue tumor located on both inside and outside the cavity of the tracheal posterior wall. In addition, the intratracheal tumor was round, and its upper margin was $2.1 \mathrm{~cm}$ away from the glottis. The length of the diseased trachea was $3.9 \mathrm{~cm}$. The corresponding tracheal cavity became narrow, and the pathological change in the surrounding tissue outside the trachea and within its boundaries was clear, without other abnormal findings; therefore, a tracheal tumor was considered ( - Fig. 1A, B). The trauma experienced by this patient was not serious, and the corresponding symptoms may have been caused by this tracheal tumor. Afterward, the patient was admitted to the respiratory department.

Subsequently, the patient underwent fiberoptic bronchoscopy and interventional therapy. The bronchoscopy showed that there was a tumor $(\sim 1.5 \mathrm{~cm}$ below the glottis) on the posterior wall of the trachea, which demonstrated an $\sim 2.3 \times 2.3 \mathrm{~cm}$ hemispherical bulge with smooth and soft surfaces; moreover, most of the tracheal cavity was occupied, and a fissure-like stenosis was formed (-Fig. 1C). An extended suction tube was placed into the distal end of the tumor to provide oxygen. After the ventilation was improved, puncture biopsy was performed by using a $14 \mathrm{G}$ needle, and then a highfrequency electrocoagulation encirclement was performed. Afterward, a new organism $(\sim 1.2 \times 1.0 \mathrm{~cm})$ was resected by a COOK AS-1 snare via high-frequency electrocoagulation, and most of the tumor tissues were removed from inside the trachea; then, a $2.0 \times 5.0 \mathrm{~cm}$ tracheal stent was placed. The upper edge of the stent was located $\sim 1.5 \mathrm{~cm}$ below the glottis, and the lower edge was $7 \mathrm{~cm}$ away from the carina of the trachea (-Fig. 2A). After this operation, the symptom of dyspnea was significantly alleviated. One week later, tracheal lipoma was confirmed by pathological examination. For this reason, the tracheal stent was removed via bronchoscopy, and the residual tumor tissues were removed as much as possible with the COOK AS-1 snare combined with high-frequency electrocoagulation. The operation was performed successfully. As the tumor tissues in the trachea were benign and resected very thoroughly, no tracheal stent was placed at this time. However, previous symptoms appeared immediately after the operation. After 6 days, the patient underwent bronchoscopy
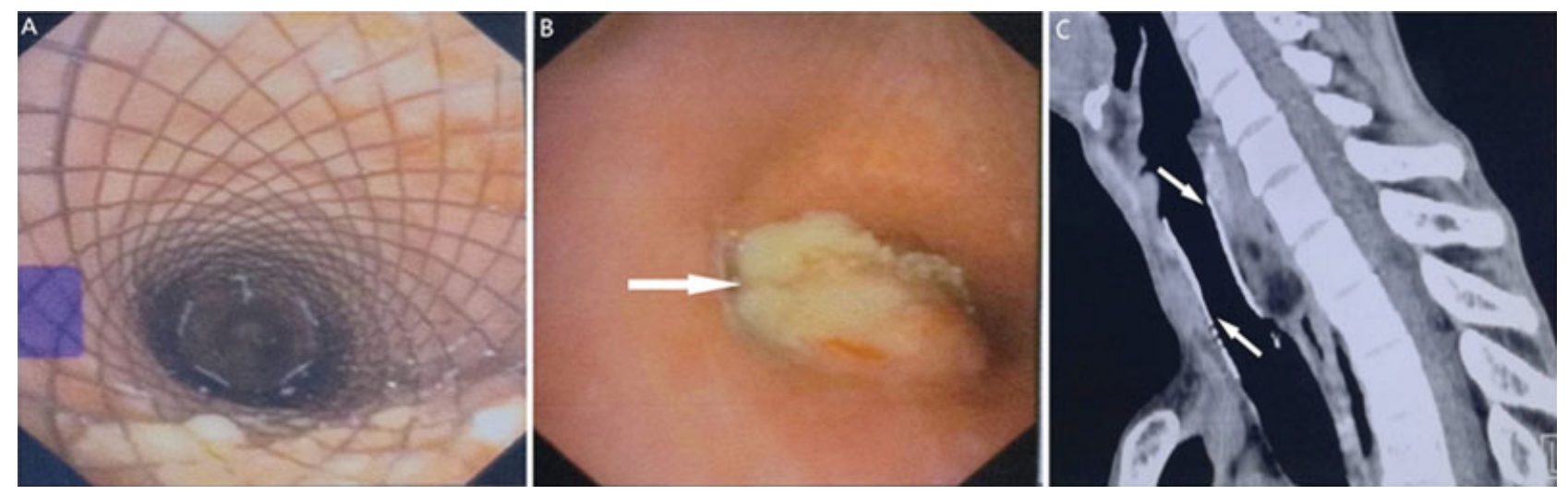

Fig. 2 (A) Bronchoscopy showing a tracheal stent placed inside the tracheal cavity. (B) Bronchoscopy showing an extratracheal tumor swelling into the trachea. (C) CT showing a tracheal stent placed again and complete patency of the stent. CT, computed tomography. 
again. A $2.0 \times 2.3 \mathrm{~cm}$ tumor $(\sim 1.5 \mathrm{~cm}$ below the glottis $)$ was found on the posterior wall of the trachea, whose surface was rough (-Fig. 2B). Therefore, an extratracheal tumor that had swelled into the trachea was considered to lead to the abovementioned symptoms. A $1.0 \times 0.8 \mathrm{~cm}$ tumor was resected with the COOK AS- 1 snare, and the tracheal stent was placed again to improve the patient's ventilation. After 4 days, chest CT showed complete patency of the stent (-Fig. 2C).

After the consultation, this patient was transferred to our hospital, and surgery was performed after placement of the tracheal stent for 10 days. After total intravenous anesthesia and orotracheal intubation, the tube was placed underneath the tumor through the tracheal stent. Using a collar-like transverse incision in the neck, under the guidance and location of bronchoscopy, the cannula placed for tracheal intubation was retracted below the glottis, and the position of the tumor was initially determined according to the photic and opacity zones on the tracheal wall, whose accurate positions were confirmed by puncture with a fine syringe needle. The trachea was cut off $\sim 0.5 \mathrm{~cm}$ below the tumorous lesion, and then the metal stent was removed; afterward, the secretions inside the lower trachea and bronchus were removed as soon as possible, and the sterile tracheal tube was inserted to ensure effective ventilation. Then, the extratracheal pathological tissues were carefully dissociated to avoid damage to surrounding tissues, such as the recurrent laryngeal nerve, blood vessels, and the esophagus. The trachea was also cut off $\sim 0.5 \mathrm{~cm}$ above the tumorous lesion, and the tumor tissue was completely removed. The length of the resected trachea was $4.9 \mathrm{~cm}$, including seven tracheal rings ( - Fig. $\mathbf{3 A}$ ). The specimen was sent for rapid biopsy, which reported a negative stump. Then, interrupted sutures were adopted with 3-0 Prolene thread, and end-to-end anastomosis of the trachea was performed. The cannula placed for tracheal intubation was removed before tying knots. Afterward, the cannula for orotracheal intubation was placed underneath the anastomotic stoma to continue respiratory support, and knots were tied under the condition of tension to decrease anterior cervical flexion. Moreover, no air leaks were found at the anastomotic stoma according to an air charging test. After that, the subcutaneous layer and skin of the patient's underjaw and anterior chest, respectively, were sutured with thick silk threads to maintain a position of anterior cervical flexion after surgery. As soon as the patient was awake, the cannula placed for tracheal intubation was removed, and he breathed naturally and smoothly. Tracheal lipoma was diagnosed according to the postoperative pathological examination ( - Fig. 3B). The mandibular and anterior chest threads were removed 3 weeks after the surgery; tracheal patency was confirmed by CT 3 months postoperatively, and no tumor was found ( - Fig. 3C). In addition, no previous symptoms, such as cough and dyspnea, appeared after the surgery.

\section{Discussion}

The majority of adult primary tracheal tumors are malignant, and benign tumors are rare. ${ }^{2,3}$ Primary benign tumors of the trachea mainly include leiomyoma, papilloma, fibroma, hemangioma, chondroma, and mixed tumors of the salivary gland, ${ }^{4,5}$ while lipoma is very uncommon.

Benign tracheal tumors tend to grow slowly and are asymptomatic at early stages. Therefore, they are difficult to be diagnosed during this period; moreover, such tumors are easily misdiagnosed after symptoms appear. Symptoms of airway obstruction appear when the degree of tracheal blockage increases to 50 to $75 \%$ or when the lumen diameter is less than $8 \mathrm{~mm} .^{1}$ Generally, it is difficult to find a lesion with conventional chest $\mathrm{X}$-ray because the trachea is covered by the mediastinum. Furthermore, due to the low morbidity of this disease, bronchial antispasmodic drugs have certain effects; therefore, these lesions are usually misdiagnosed as bronchial asthma or pulmonary infection. This patient had a chronic history lasting for 4 years and had been misdiagnosed with bronchial asthma during that time. Because of the trauma, the diagnosis was able to be made through cervical, thoracic CT and 3D reconstruction. This case suggests that in patients with dyspnea, which worsens gradually, the possibility of a tracheal tumor should be considered, and timely examination, including cervical, thoracic CT and bronchoscopy, can provide an accurate diagnosis. In fact, the physical sign of this disease is mainly inspiratory dyspnea, and it is evidently different from expiratory dyspnea caused by bronchial asthma and pulmonary infectious diseases. Careful physical examination can be helpful for differential diagnosis.
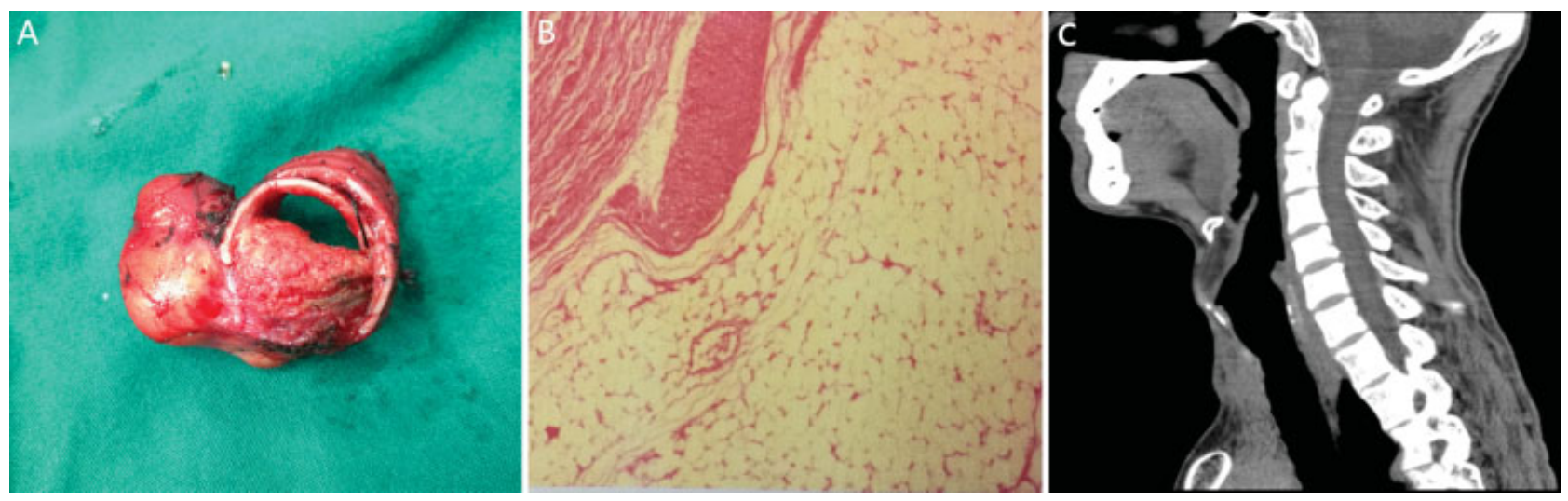

Fig. 3 (A) Macrography showing a resected tracheal tumor and rings. (B) Pathological examination confirming a tracheal lipoma. (C) Postoperative CT showing tracheal patency and no tumor. CT, computed tomography. 
In this case, this patient has suffered endobronchial interventions four times, and a stent was implanted at the first and third times. That is because the respiratory physician worried about the resection of the tumor was incomplete, and there could be some of the residual tumors which may lead to a stenosis of the trachea. In addition, we have described a puncture biopsy of the tumor during the first endobronchial intervention, whereas it showed a negative result. Soon, most of the tumors have been resected and the ultimate tracheal lipoma was reported by pathological examination. Thus, a fresh-frozen examination of the tumor was not necessary during our final surgical treatment.

However, there are some limitations to the therapeutic process. As is known, various interventional treatments via fiberoptic bronchoscopy should only be applied to narrowbased benign tracheal tumors that originate from submucosal, nodular, or polypoid tissues protruding into the tracheal lumen, where the base is connected to the tracheal wall by a pedicle with irregular length and uneven thickness. Moreover, the placement of a tracheal stent may lead to a secondary obstruction caused by the growth of the tracheal tumor, such as tracheal secretions blocking the distal end of the stent, bleeding, and infection; the placement of a stent can only alleviate the symptoms of airway obstruction to some extent and cannot achieve a cure or prolong survival time in patients. Thus, a stent is only used for patients without the opportunity for surgical therapy, who are not suitable for surgical resection, or who seek relief from acute airway obstruction as well as for saving rescue time.

In this patient, $\mathrm{CT}$ and $3 \mathrm{D}$ reconstruction of the neck and chest showed a soft tissue tumor located on both inside and outside the cavity of the tracheal posterior wall. Afterward, high-frequency electrocoagulation and placement of a tracheal stent were first performed via bronchoscopy. After the symptom of dyspnea improved, surgery was performed immediately, especially because pathological biopsy reported a benign tracheal lipoma. Since then, multiple interventions with bronchoscopy and placements of a tracheal stent have resulted in repeated respiratory symptoms repeatedly, increased patient suffering and risk, and prolonged curative time.

In this case, the diameter of the tracheal wall invaded by this tumor was $\sim 70 \%$, and the length of the tracheal lesion was $3.9 \mathrm{~cm}$. The boundary between the extratracheal lesion and the surrounding tissue was distinct, and such lesions are resectable. The preferred surgical procedure is sleeve resection and end-to-end anastomosis of the trachea.

According to the preoperative findings from CT and bronchoscopy, as well as the pathological data, we paid attention to the following points during the surgery. (1) As the tracheal lesion was long, on the basis of complete resection of the tumor, the normal tracheal ring was retained as much as possible to reduce the tension from anastomosis. The extent of tumor invasion under the mucosa was wider than what we observed in the intracavity of the trachea with the naked eye; although this patient had a benign lipoma, to achieve the purpose of radical resection, the tracheal margin was made $\sim 0.5 \mathrm{~cm}$ away from the upper and lower edges of the tumor.
Therefore, under the guidance of bronchoscopy during the operation, tracheal penetration with a syringe needle was used to determine, as accurately as possible, the tracheal margin needed for resection. (2) Although the resected trachea was long, to avoid excessive dissociation of the tracheal tissue, which may impair the blood supply of the anastomosis, we considered whether it was sufficient to dissociate the upper and lower ends of the trachea before achieving anastomosis. (3) During dissociation of the extratracheal tumor, damage to the recurrent laryngeal nerve, blood vessels, the esophagus, and other surrounding tissue should be avoided. (4) To reduce anastomotic tension during the operation and postoperation, knots were tied in anterior cervical flexion and the subcutaneous layer and skin of the patient's underjaw and anterior chest, respectively, were sutured with thick silk threads to maintain anterior cervical flexion after surgery. The threads were removed 3 weeks after surgery, and the back of the head was avoided for more than 3 months.

\section{Conclusion}

Primary tracheal tumors should be highly suspected in patients with recurrent and gradually worsening dyspnea symptoms, including the three depressive signs of wheezing in the neck, upper chest, and chest; timely cervical, thoracic CT and fiberoptic bronchoscopy can provide an accurate diagnosis.

Various interventional treatments via fiberoptic bronchoscopy are mainly suitable for benign tracheal tumors with narrow margins, and tracheal stent placement can only alleviate the patient's symptoms of airway obstruction to some extent. Surgical radical resection is the only way to cure all benign tracheal tumors.

If the circumference of the tracheal wall is mostly invaded by the tracheal tumor and the length of the tracheal lesion is long, the preferred surgical procedure should be sleeve resection and end-to-end anastomosis of the trachea.

\section{Conflict of Interest}

All the authors have read the article and approved its submission. The authors report no conflict of interest. Written informed consent was obtained from the patient for publication of this case report and the accompanying images. The identity of the patient has been protected.

\section{References}

1 Macchiarini P. Primary tracheal tumours. Lancet Oncol 2006;7 (01):83-91

2 Grillo HC. Primary tracheal tumours. In: Grillo HC, ed. Surgery of the Trachea and Bronchi. Hamilton, London: BC Decker; 2004: 208-247

3 Junker K. Pathology of tracheal tumors. Thorac Surg Clin 2014;24 (01):7-11

4 Beheshti J, Mark EJ, Graeme-Cook F. Epithelial tumor of the trachea. In: Grillo HC, ed. Surgery of the Trachea and Bronchi. Hamilton, London: BC Decker; 2004:73-85

5 Beheshti J, Mark EJ. Mesenchymal tumor of the trachea. In: Grillo HC, ed. Surgery of the Trachea and Bronchi. Hamilton, London: BC Decker; 2004:86-97 